\title{
INTERAÇÃO ENTRE ANTICONCEPCIONAIS ORAIS COM ANTIBIÓTICOS: UMA REVISÃO INTEGRATIVA
}

\author{
INTERACTION BETWEEN ORAL CONTRACEPTIVES WITH ANTIBIOTICS: AN \\ INTEGRATIVE REVIEW
}

\author{
Andrey Gonçalves de Aquino ${ }^{1}$ \\ Davy Gonçalves Rios ${ }^{2}$ \\ Marcos Paulo Santos Passos ${ }^{3}$
}

RESUMO: Este estudo teve como objetivo analisar na literatura científica sobre a relação na interação medicamentosa entre antibióticos e anticoncepcionais. Foi feito uma revisão sistemática da literatura em diversas bases de dados, com o intervalo temporal entre $2008 \mathrm{e}$ 202I. Anticoncepcionais orais são o método mais difundido e usado no mundo e considerado um método reversível muito eficaz, já os antibióticos são compostos naturais ou sintéticos capazes de inibir o crescimento, ou causar a morte bactérias. A interação medicamentosa desses dois fármacos se dá principalmente por dois motivos, o primeiro deles é causando a morte das bactérias do cólon, as quais são fundamentais para a farmacocinética dos contraceptivos orais, e o segundo é acelerando a biotransformação, diminuindo a concentração sérica, reduzindo a eficácia e requerendo ajuste na dosagem de diversas drogas metabolizadas no fígado.

Palavras-chave: Anticoncepcionais. Antibióticos. Interação.

ABSTRACT: This study aimed to analyze the scientific literature on the relationship in drug interaction between antibiotics and contraceptives. A systematic literature review was carried out in several databases, with the time interval between 2008 and 202I. Oral contraceptives are the most widespread and used method in the world and considered a very effective reversible method, as antibiotics are capable natural or synthetic compounds of inhibiting the growth or causing death of bacteria. The drug interaction of these two drugs occurs mainly for two reasons, the first is causing the death of colon bacteria, which are

\footnotetext{
I Universidade Salvador -UNIFACS, Bahia, Brasil.E-mail: andrey.goncalves2o2i@outlook.com, https://orcid.org/oooo-0oo2-2060-733I

${ }_{2}$ Universidade Salvador -UNIFACS, Bahia, Brasil.E-mail: davyrios25@gmail.com, https://orcid.org/oooo$0002-7458-7436$

${ }^{3}$ Especialista em análises clínicas e citopatologia clínica. Universidade Salvador- UNIFACS. ORCID: https://orcid.org/oooo-ooor-9076-4650
} 
fundamental for the pharmacokinetics of oral contraceptives, and the second is accelerating the biotransformation, decreasing the serum concentration, reducing effectiveness and requiring adjustment in the dosage of several drugs metabolized in the liver.

Keywords: Contraceptive. Antibiotics. Interaction.

\section{INTRODUÇÃO}

Os contraceptivos orais são formados pelos hormônios estrogênio e progesterona, que atuam na inibição da ovulação, atrofiando o revestimento do útero e dificultando a passagem dos espermatozoides devido ao aumento da viscosidade do muco cervical. Para diminuir os riscos cardiovasculares e outros efeitos colaterais associados às pílulas, as dosagens hormonais desses remédios são bastante reduzidas. Na presença de antibióticos, os níveis dos hormônios que já são reduzidos, podem cair ainda mais, comprometendo a eficácia dos anticoncepcionais orais (MENDONÇA, 2017).

As interações medicamentosas acontecem quando o efeito de um medicamento é alterado pela presença de outro. A administração concomitante entre dois fármacos pode levar ao antagonismo, sinergismo ou potencialismo destas substâncias, elevando ou reduzindo a toxicidade e/ou efeito terapêutico. Um relato de interação medicamentosa é entre os anticoncepcionais orais e alguns antibióticos, pois, o uso concomitantemente da pílula anticoncepcional com antibióticos pode não fazer efeito desejado ao método contraceptivo devido à interação existente entre essas duas classes de medicamentos, elas podem interagir entre si, e causar uma diminuição do efeito terapêutico dos anticoncepcionais, consequentemente, continuando a ovulação, expondo as mulheres ao risco de gravidez indesejada (MARTINS, 2014).

Existem diversos medicamentos que podem reduzir da eficácia contraceptiva. Os grandes vilões dos anticoncepcionais sãos os antibióticos como Amoxicilina e Rifampicina, pois, modificam a absorção intestinal dos contraceptivos orais, influenciando diretamente na ação dos mesmos através do sinergismo, termo utilizado para designar a potencialização do efeito do medicamento, ou inibindo o efeito do medicamento (TURCATO, 2016).

Segundo o Ministério da Saúde, o SUS disponibiliza oito métodos contraceptivos 


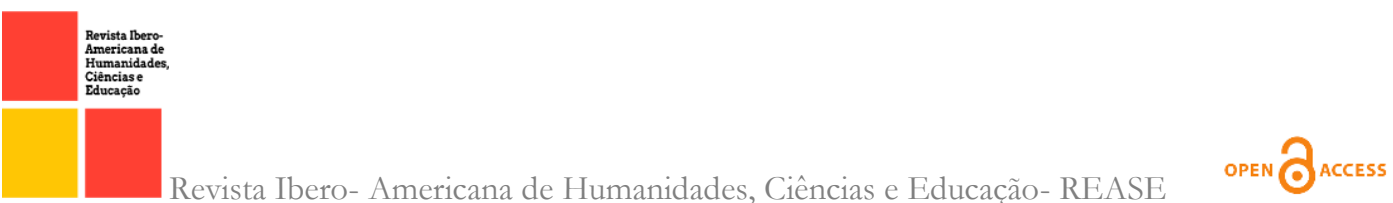

diferentes, os quais são: métodos naturais; métodos hormonais; métodos de barreira; dispositivo intrauterino (DIU) de cobre ou com hormônios; contracepção de emergência e métodos definitivos (RAMOS, 2008).

Dentre todos os métodos contraceptivos existentes, os anticoncepcionais orais (AO) são os que mais se destacam, e o que mais são procurados pelas mulheres. Os anticoncepcionais orais podem ser combinados (estrógenos + progesterona), mono, bi e trifásicos, apresentando eficácia de 99,9\% e efetividade de $97-98 \%$. O mecanismo de ação dos anticoncepcionais acontece de diversas formas, sendo elas a inibição da ovulação, aumentando a produção de muco cervical para impedir a passagem dos espermatozoides e atrofiando o revestimento do útero (MENDONÇA, 2017).

Considerando a extensa utilização destes agentes farmacológicos em ocasiões de administração simultânea, o presente estudo tem o propósito de analisar a existência ou não de interação medicamentosa entre hormônios contraceptivos femininos e fármacos antibióticos e os possíveis mecanismos que levam essa interação. Material e Métodos

O trabalho desenvolvido caracteriza-se como revisão sistemática da literatura. As informações contidas designam-se de artigos científicos que abordam a temática "Interação medicamentosa entre antibióticos e anticoncepcionais, seu mecanismo de ação, seus riscos e consequências", baseando-se em uma análise crítica da literatura em artigos e revistas científicas. O trabalho foi desenvolvido a partir de artigos e revistas nacionais e internacionais, onde todos se encontram disponíveis "online" em texto completo.

As referências apresentadas serão coletadas a partir das bases de dados MedLine, PubMed, ScienceDirect e LILACS (Literatura Latino-Americana e do Caribe em Ciências da Saúde), utilizando AND como operador booleano e tendo as seguintes palavras-chave: "Contraceptive" e "Antibiotics".

Foram incluídos também conteúdos em português e inglês. Os critérios de exclusão serão: duplicidade, incompatibilidade com o tema e artigos de revisão. O período de busca será de artigos publicados nos últimos 13 anos (2008 a 2021). 


\section{RESULTADOS}

Conforme a análise inicial dos estudos, foi identificado um total de 1257 publicações, sendo encontrados 634 no MedLine, 553 no ScienceDirect, 45 no PubMed e 25 no LILACS.

$\mathrm{Na}$ base de dados MedLine foi utilizado as palavras chaves "antibiótics" e “contraceptives”, o número de artigos foram reduzidos de 634 para 139 após a filtragem de “ano". No ScienceDirect foram utilizadas as palavras chaves "Antibiotics", "Oral Contraceptives" e "Interation", sendo apresentados 553 artigos no período temporal especificado. No PubMed foram encontrados 45 publicações sendo utilizadas "Antibiotics" e "Contraceptives" como palavras chaves e os seguintes filtros "ano", "texto-completo", "línguas - português e inglês" e "categoria de artigos - ensaio clínico". No LILACAS foram encontrados 25 artigos com as seguintes palavras chaves "Antibiótics" e “Contraceptives”, porém reduziu para 9 publicações, as quais estavam na faixa temporal.

Após a utilização dos filtros, dos 1257 (publicações) encontradas, foram removidos 514 por faixa temporal e 5 por duplicata. Dos 738 artigos incluídos relacionados para análise de títulos, a leitura feita por dois avaliadores, com posterior discussão em relação a possíveis divergências, de modo a buscar um consenso, totalizando 723 artigos excluídos por serem artigos de revisão ou apresentarem incompatibilidade com o tema. Restando apenas is artigos selecionados para leitura dos resumos. Dos 15 artigos destinados à leitura dos resumos, 4 foram excluídos por critérios de exclusão, onde, apenas in foram selecionados para serem lidos na íntegra. Das ir publicações lidas na íntegra, apenas 4 foram excluídas por critérios de exclusão, totalizando em 7 artigos incluídos para compor a revisão sistemática. O destalhamento dos processos de identificação, seleção e inclusão estão apontados no fluxograma da Figura I. 
Neste estudo pode-se observar que dentre todas as pesquisas feitas utilizando diferentes antibióticos de amplo espectro, com anticoncepcionais combinados, não foi possível identificas alterações significativamente relativas que possam alterar os níveis de hormônios em mulheres que fazem o uso dessa combinação. Os dados obtidos com o levantamento dos estudos são mostrados na Tabela I.

Figura I - Fluxograma de seleção de estudos

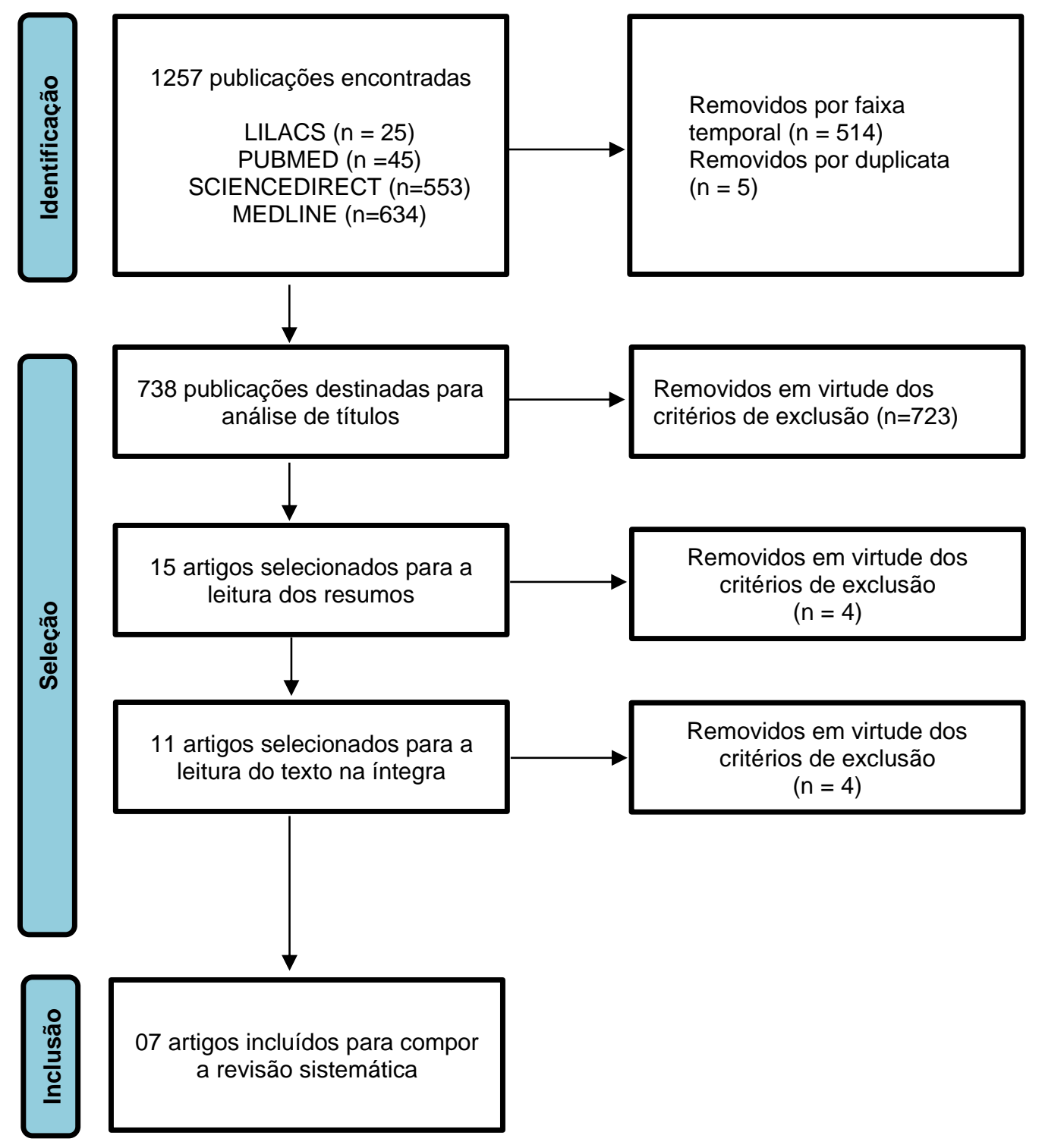




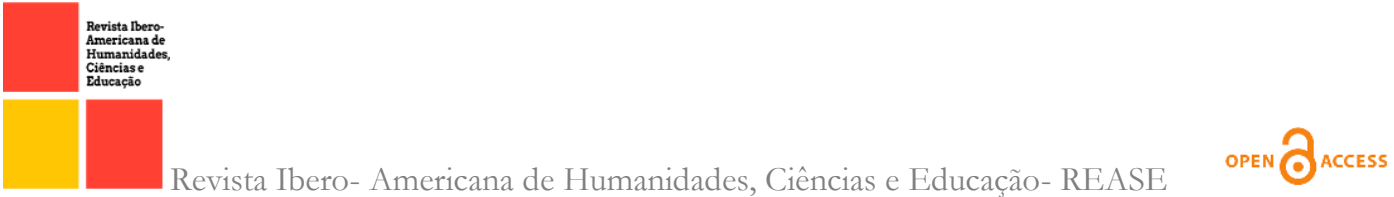

Fonte: Adaptado do PRISMA flow diagram, 2020 
TABELA r. Apresentação da síntese dos artigos selecionados e analisados na revisão, segundo autor, ano, antibiótico abordado no estudo, contraceptivos orais estudados, mecanismo de interação desobertos e os desfechos dessas interações.

\begin{tabular}{|c|c|c|c|c|}
\hline $\begin{array}{l}\text { Blode et al,. } \\
2012\end{array}$ & Rifampicina; & $\begin{array}{l}\text { Anticoncepcional } \\
\text { combinado }\end{array}$ & $\begin{array}{c}\text { Indutores } \\
\text { da } \\
\mathrm{CYP}_{3} \mathrm{~A}_{4}\end{array}$ & $\begin{array}{l}\text { A rifampicina afeta a } \\
\text { eficácia contraceptiva }\end{array}$ \\
\hline $\begin{array}{c}\text { J Taylor; } M \\
\text { N } \\
\text { Pemberton,. } \\
2012\end{array}$ & Penicilina; & $\begin{array}{c}\text { Anticoncepcional } \\
\text { combinado }\end{array}$ & $\begin{array}{l}\text { Reduzem } \\
\text { as } \\
\text { bactérias } \\
\text { do cólon }\end{array}$ & Não houve interação \\
\hline $\begin{array}{l}\text { Koopmans } \\
\text { et al,. } 2012\end{array}$ & $\begin{array}{l}\text { Trimetropina; } \\
\text { Amoxicilina; } \\
\text { Nitrofurantoína; } \\
\text { Doxiciclina. }\end{array}$ & $\begin{array}{l}\text { Anticoncepcional } \\
\text { combinado }\end{array}$ & $\begin{array}{l}\text { Reduzem } \\
\text { as } \\
\text { bactérias } \\
\text { do cólon }\end{array}$ & Fraca chance de falha \\
\hline $\begin{array}{l}\text { Masters } \\
\text { KP, Carr } \\
\text { BM,. } 2009\end{array}$ & $\begin{array}{l}\text { Amoxicilina; } \\
\text { Tetraciclina; }\end{array}$ & $\begin{array}{l}\text { Anticoncepcional } \\
\text { combinado }\end{array}$ & $\begin{array}{l}\text { Reduzem } \\
\text { as } \\
\text { bactérias } \\
\text { do cólon }\end{array}$ & $\begin{array}{l}\text { Não houve diminuição dos } \\
\text { esteróides plasmáticos }\end{array}$ \\
\hline $\begin{array}{c}\text { Pottegard et } \\
\text { al,. 2018 }\end{array}$ & Dicloxacilina & $\begin{array}{l}\text { Anticoncepcional } \\
\text { combinado }\end{array}$ & $\begin{array}{l}\text { Indutor } \\
\mathrm{da} \\
\mathrm{CYP}_{3} \mathrm{~A}_{4}\end{array}$ & $\begin{array}{c}\text { Não houve evidências para } \\
\text { um risco de falha }\end{array}$ \\
\hline $\begin{array}{l}\text { Toh et al,. } \\
\text { 2oII }\end{array}$ & $\begin{array}{l}\text { Amoxicilina; } \\
\text { Ampicilina; }\end{array}$ & $\begin{array}{l}\text { Anticoncepcional } \\
\text { combinado }\end{array}$ & $\begin{array}{l}\text { Reduzem } \\
\quad \text { as } \\
\text { bactérias } \\
\text { do cólon }\end{array}$ & $\begin{array}{l}\text { Não foi encontrado } \\
\text { associação entre o uso de } \\
\text { antibióticos com } \\
\text { anticoncepcionais. }\end{array}$ \\
\hline $\begin{array}{l}\text { Wiesinger } \\
\text { et al,. 2020 }\end{array}$ & Rifampicina; & $\begin{array}{l}\text { Anticoncepcional } \\
\text { combinado }\end{array}$ & $\begin{array}{l}\text { Indutores } \\
\text { da } \\
\mathrm{CYP}_{3} \mathrm{~A}_{4}\end{array}$ & $\begin{array}{l}\text { A rifampicina se mostrou } \\
\text { como forte indutor da } \\
\mathrm{CYP}_{3} \mathrm{~A}_{4} \text {, diminuindo os } \\
\text { níveis de hormônios nas } \\
\text { pacientes. }\end{array}$ \\
\hline
\end{tabular}




\section{DISCUSSÃO}

Depois da análise dos resultados, notou-se que os artigos encontrados abordam sobre a temática proposta, e mesmo as publicações que não estavam relacionados diretamente ao tema abordado no projeto, contribuíram bastante com a revisão sistemática integrativa. Nesse contexto foram encontrados dois mecanismos explicando como ocorrem as possíveis interações entre anticoncepcionais orais e antibióticos. Mas antes de apresentar as interações existentes ou não, é necessário compreender mais sobre os anticoncepcionais orais.

Existem dois tipos principais de contracepção hormonal. Estes são o anticoncepcional hormonal combinado (C.O.C.) e o anticoncepcional só de progestagênio. A contracepção hormonal combinada contém um componente estrogênio e um progestogênio. Quando a quantidade de estrogênio e progestogênio é fixa, eles são conhecidos como "monofásicos". Quando a quantidade de hormônio varia conforme o estágio do ciclo, eles são conhecidos como "fásicos". As pílulas fornecem contracepção principalmente pela supressão do hormônio folículo-estimulante (FSH) e do hormônio luteinizante (LH), inibindo assim o crescimento e desenvolvimento folicular e a ovulação. Os efeitos secundários no muco cervical e na histologia endometrial que reduzem a probabilidade de fertilização ou implantação também podem contribuir. Produtos só de progestógeno ("minipílulas”) inibem a ovulação em menos de 50\% das usuárias, fornecendo contracepção principalmente pelo (espessamento) do muco cervical e manutenção de um ambiente endometrial inadequado para implantação e nutrição de um óvulo fertilizado (BARRY,. ET AL 200I).

Os mecanismos de interação dos C.O.C. com antibióticos acontecem por meio dos antibióticos indutores e não indutores de enzimas. A interação com antibióticos indutores acontece da seguinte forma, o etinilestradiol oral é absorvido no intestino delgado e sofre metabolismo de primeira passagem no fígado. Uma vez conjugado com o ácido glucurônico, é excretado na bile e passa para o intestino delgado e depois para o intestino grosso. As enzimas hidrolíticas produzidas por bactérias no intestino grosso fragmentam os conjugados de etinilestradiol, que os liberam para serem reabsorvidos no intestino grosso. Estes, então, entram na circulação entero-hepática, a enzima hepática o citocromo p-45o acelera o metabolismo do etinilestradiol, o que leva a níveis menos circulantes. Uma redução nos 
níveis séricos de etinilestradiol pode levar à redução da eficácia da contracepção. Já quando administrados com antibióticos não indutores de enzimas, os contraceptivos orais podem reduzir as bactérias do cólon, portanto, reduzir teoricamente a circulação entero-hepática de etinilestradiol. Isso pode levar à redução da eficácia da contracepção oral combinada e resultar em gravidez não planejada. (J TAYLOR; M N PEMBERTON,. 2012)

No primeiro artigo estudado, escrito pelos autores Koopmans et al., foi realizada uma pesquisa na base de dados (IADB) a qual contém registro de dispensação de medicamentos na Holanda. Nessa base de dados foram encontrados: medicamentos dispensados, data da prescrição e posologia. No IADB foi gerado um banco de dados de gravidez, onde foram selecionadas todas as mães entre is e 49 anos, que deram à luz uma criança entre janeiro de 1995 e setembro 2009. Após isso, 397 mulheres foram selecionadas por fazerem o uso de anticoncepcionais orais combinados (C.O.C.) e antibióticos de amplo espectro, especificadamente, trimetoprima, amoxicilina, nitrofurantoína, doxiciclina, feneticilina, claritromicina e fenoximetilpenicilina. No final da pesquisa, os resultados encontrados foram inconclusivos. É possível que ocorra uma interação entre COs e antibióticos em um pequeno subgrupo de mulheres com níveis incomuns de hormônio esteroide baixo, taxas relativamente altas de conjugação hepática ou intestino flora particularmente suscetível aos antibióticos sendo usados, mas sugerem uma relação fraca entre o uso de antibióticos e falha de C.O.C. (KOOPMANS ET AL, 2012)

Um estudo feito por Toh et al, nos Estados Unidos relatou que 1.330 mulheres que usavam COCs relataram que a gestação não foi planejada, assim casos de falha de anticoncepcional, a prevalência de uso de antibióticos foi de 3,6\% durante as 4 semanas antes da data da concepção e 3,9\% durante as $4-8$ semanas anteriores à data de concepção, assim, mais de $80 \%$ dos antibióticos tiveram uma duração de tratamento de 14 dias ou menos. A amoxicilina e a ampicilina foram os antibióticos mais usados, seguidos dos macrolídios e das sulfonamidas.

Quando os anticoncepcionais orais são ingeridos, o estrógeno e a progesterona são rapidamente absorvidos no trato gastrintestinal, caindo prontamente na corrente sanguínea $e$ conduzidos até o fígado, onde são metabolizados e formam conjugados inativos. De todo composto metabolizado, apenas uma pequena parte é hidrolisada pelas enzimas das bactérias intestinais, liberando o estrógeno ativo, então o mesmo é reabsorvido, e estabelece o ciclo 
entero-hepático que aumenta o nível plasmático de estrógeno circulante (CORREA;ANDRADE; RANALLI,1998).

Antibióticos de amplo espectro como amoxicilina, podem perturbar a flora bacteriana intestinal, levando a uma diminuição na reabsorção intestinal de COCs e uma redução consequente nos níveis circulantes de COCs necessários para alcançar a contracepção, porém os estudos farmacocinéticos não parecem apoiar um efeito dos antibióticos nos níveis plasmáticos de COCs (exceto rifampicina), mas uma série de estudos sugeriram que algumas mulheres podem ser mais suscetíveis a uma diminuição induzida por antibióticos na concentração plasmática de COCs (TOH ET AL, 20II).

De acordo com Toh et al, não foi encontrado uma associação entre o uso concomitante de antibióticos e o risco de gravidez entre usuárias de COCs, porém não pode ser descartado um risco aumentado de falha do COCs com o uso concomitante de antibióticos, devido aos conhecimentos farmacocinéticos e farmacodinâmicos do efeito limitado dos antibióticos no metabolismo de esteróides contraceptivos.

Conforme Taylor et al., em 201 no País de Gales 8 um estudo publicado no British Dental Journal, mostrou que $67 \%$ dos antibióticos prescritos por dentistas eram penicilinas, seguidos por clindamicina, macrolídios e tetraciclinas, sendo todos não indutores de enzimas. Recentemente, houve uma mudança, baseado em evidências que analisam as interações de antibióticos não indutores de enzimas. Vários estudos e ensaios examinaram os níveis de etinilestradiol em pacientes que tomavam a pílula anticoncepcional oral combinada (COCs) e antibióticos e não encontraram níveis diminuídos de etinilestradiol ou qualquer alteração na farmacocinética do etinilestradiol. Com base nos resultados encontrados no estudo, é recomendado que ao prescrever antibióticos não indutores de enzimas para pacientes que usam contracepção hormonal combinada, a orientação atual é não haver necessidade de dizer aos pacientes que eles devem usar métodos anticoncepcionais adicionais enquanto tomam os antibióticos. (J TAYLOR; M N PEMBERTON,. 2012)

Uma pesquisa feita em 2009 nos Estados Unidos, visando avaliar o conhecimento dos médicos e farmacêuticos sobre as potenciais interações medicamentosas entre os anticoncepcionais orais combinados (C.O.C.) e os antibióticos de amplo espectro determinou que as mulheres que tomam antibióticos orais concomitantes podem causar uma redução do etinilestradiol e das progestinas. Os participantes do estudo acreditam que os antibióticos de 
amplo espectro diminuem a eficácia dos COCs, pois reduzirem as bactérias do cólon e, assim, prevenir a conversão dos conjugados de etinilestradiol em metabólitos ativos que são reabsorvidos. Porém, o risco de gravidez pela interação entre esses antibióticos com os contraceptivos é muito baixo, praticamente nulo, mas comumente os médicos alertam e para a paciente se sentir mais segura indicam um método contraceptivos alternativo (MASTERS KP, CARR BM, 2009).

Além da redução das bactérias do colón, existe outro mecanismo onde os antibióticos parecem reduzir os níveis plasmáticos dos hormônios contraceptivos que é a indução das enzimas do citocromo $\mathrm{P} 450$ no fígado, onde essas enzimas irão acelerar o metabolismo dos anticoncepcionais. Entre os antibióticos estudados por esse mecanismo de ação, a rifampicina é o principal fármaco responsável pelo maior número de casos de ineficácia dos anticoncepcionais (CORRÊA, ET AL., 1998).

Segundo Blode et al,. Foi realizado um estudo onde foram avaliados os efeitos da indução e inibição do citocromo $\mathrm{P}_{450}{ }_{3} \mathrm{~A}_{4}\left(\mathrm{CYP}_{3} \mathrm{~A}_{4}\right)$ na farmacocinética de estado estacionário dos componentes de um novo contraceptivo oral (ACO) contendo valerato de estradiol ( $\left.E_{2} V\right)$ e dienogeste (DNG).

Em relação à interação medicamentosa dos anticoncepcionais, destaca-se aquelas onde há a possibilidade de indução enzimática relacionada com o citocromo $\mathrm{P}-450 \mathrm{CY} \mathrm{P}_{3} \mathrm{~A}_{4}$, subfamília que possui especificidade por compostos lipofílicos, incluindo esteroides. Visto que tais enzimas são induzidas por outros medicamentos, a metabolização dos hormônios pode se tornar mais rápida e extensa (TURCATO ET AL,. 2017).

Dois estudos foram, portanto, realizados para investigar os efeitos da indução e inibição do $\mathrm{CYP}_{3} \mathrm{~A}_{4}$ no equilíbrio perfil farmacocinéticos estaduais de DNG, E2, estrona (EI) e sulfato de estrona (EI-S) após a administração de valerato de estradiol e dienogeste em combinação. Os dois estudos foram realizados conforme a Declaração de Helsinque e as Diretrizes ICH-GCP. O primeiro estudo foi de centro único, aberto, não randomizado projetado para investigar o efeito da indução do $\mathrm{CYP}_{3} \mathrm{~A}_{4}$ pela rifampicina na farmacocinética de estado estacionário de estradiol e dienogeste em i6 mulheres pós-menopáusicas saudáveis. O Estudo 2 foi um estudo aberto, de grupo paralelo, cruzado unilateral, projetado para investigar o efeito da inibição do $\mathrm{CYP}_{3} \mathrm{~A}_{4}$ pelo cetoconazol (Grupo I) ou eritromicina (Grupo 2) na farmacocinética de estado estacionário de estradiol e dienogeste em 24 mulheres pós- 
menopáusicas saudáveis ( 12 mulheres por grupo). Ao fim da pesquisa foram obtidos os resultados onde a administração concomitante de rifampicina diminuiu a exposição sistêmica ao medicamento e produziu razões geométricas médias para estradiol concentração sérica máxima e concentração sérica-tempo entre $0-24 \mathrm{~h}$ de $75 \%$ e $56 \%$, respectivamente. As proporções médias correspondentes para dienogeste foram $48 \%$ e $17 \%$, respectivamente. A coadministração de cetoconazol aumentou a exposição sistêmica ao fármaco e gerou taxas de estradiol de $165 \%$ e $157 \%$, respectivamente, e taxas de dienogeste de $194 \%$ e $286 \%$, respectivamente. A co-administração de eritromicina também resultou no aumento da média concentração sérica máxima e concentração sérica-tempo entre o-24h de estradiol e dienogeste. As proporções médias geométricas de concentração sérica máxima e concentração sérica-tempo entre $0-24 \mathrm{~h}$ para estradiol foram de $151 \%$ e $133 \%$, respectivamente. As taxas correspondentes para dienogeste foram $133 \%$ e $162 \%$, respectivamente. Por fim, interações medicamentosas significativas são aparentes quando os moduladores do $\mathrm{CYP}_{3} \mathrm{~A}_{4}$ são coadministrados com os componentes de um contraceptivo oral contendo valerato de estradiol / dienogeste. A co-administração de moduladores do $\mathrm{CYP}_{3} \mathrm{~A}_{4}$ deve ser evitada sempre que possível, e outra categoria de contracepção deve ser usado quando a coadministração de indutores do $\mathrm{CYP}_{3} \mathrm{~A}_{4}$ como a rifampicina for inevitável (BLODE ET AL, 2012).

Conforme Wiesinger et al., foi realizado um estudo cruzado, randomizado, aberto, de sequência fixa feitas em cinco grupos conforme tenham tratamentos semelhantes em dois centros na Alemanha com o intuito de investigar efeitos da indução do $\mathrm{CYP}_{3} \mathrm{~A}$ na farmacocinética de progestágenos e etinilestradiol, o indutor escolhido para o estudo foi o antibiótico Rifampicina. Estudo este realizado em mulheres saudáveis na pós-menopausa. Para serem elegíveis, os sujeitos deveriam ter entre 45 e 70 anos (inclusive), ser não fumantes e ter peso corporal $\geq 50 \mathrm{~kg}$ e índice de massa corporal $\geq 18,5$ e $\leq 30 \mathrm{~kg} / \mathrm{m} 2$. Seu estado de pósmenopausa teve que ser confirmado pela história médica (menopausa natural $\geq 12$ meses antes da primeira dose da medicação do estudo ou ovariectomia bilateral $\geq 3$ meses antes da primeira dose) ou, para mulheres com menos de 6o anos, por medidas hormonais (hormônio folículo estimulante> $40 \mathrm{UI} / \mathrm{L}$ e estradiol $\leq 20 \mathrm{pg} / \mathrm{mL}$ ). Mulheres com riscos específicos ou com condições, ou hábitos suspeitos de afetar os objetivos do estudo foram excluídas da participação (WIESINGER, ET AL,. 2020). 
A indução enzimática também pode associar-se ao aumento da toxicidade no caso de fármacos metabolizados em formas reacionais. Em alguns casos, determinado composto pode induzir a biotransformação de outros e o seu próprio metabolismo. A inibição enzimática, no que lhe concerne, resulta em níveis elevados do fármaco original, efeitos farmacológicos prolongados e maior incidência de toxicidade da droga. Exemplo de interação medicamentosa é a indução potente de $\mathrm{CYP}_{3} \mathrm{~A}_{4}$ intestinal e hepática pela rifamicina, que determina aumento significativo na depuração de corticosteroides, diazepam, anticoncepcionais orais, etc. Além disso, as diferenças genéticas (polimorfismo genético) na capacidade das pessoas de metabolizar um fármaco através de determinada via são as responsáveis pelas grandes diferenças interindividuais na biotransformação observada numa população. As diferenças fenotípicas na quantidade da droga excretada através de uma via polimorficamente controlada levam à classificação dos indivíduos em metabolizadores rápidos ou lentos (BENET LZ ET AL,. 1996).

Ao final do estudo, foram obtidos os resultados que apontavam a indução fraca do $\mathrm{CYP}_{3} \mathrm{~A}$, confirmada por uma diminuição média na exposição ao midazolam em $46 \%$, resultou em pequenas alterações na exposição à progestina (diminuições médias: 15-37\%). A forte indução do $\mathrm{CYP}_{3} \mathrm{~A}$, em contraste, resultou em reduções médias de 57-90\% (redução média na exposição ao midazolam: 86\%). Nomeadamente, a magnitude dos efeitos de indução observados variou de fraco a forte (WIESINGER, ET AL,. 2020).

Em um estudo realizado por Pottegård et al,. Foi desenvolvido um estudo do tipo casecross-over (técnica particularmente adequada para exposições transitórias.) Com o intuito de investigar se o uso de dicloxacilina e sua indução das principais enzimas do citocromo $\mathrm{P}_{450}$ confere um risco aumentado de gravidez indesejada entre usuárias de anticoncepcionais orais.

Neste estudo, foi obtido dados do Registro de Abortos Induzidos Legalmente, o Registro Médico de Nascimento (Bliddal et al,.), o Registro Nacional de Prescrições Dinamarquês e o Registro de Pacientes Dinamarquês. A população do estudo incluiu mulheres dinamarquesas que engravidaram (1997 - 2015) durante o uso de contraceptivo oral, definido como tendo preenchido uma receita de um contraceptivo oral dentro de 120 dias antes e depois da data estimada de concepção. Entre 364 mulheres que usam dicloxacilina antes da concepção, 40 (II\%) foram expostos à dicloxacilina no momento da concepção, produzindo uma razão de possibilidades (OR) associando o uso de dicloxacilina à gravidez 
indesejada de I,I8 (Intervalo de confiança - IC 95\% 0,84-I,65). As análises suplementares e de sensibilidade geralmente retornaram estimativas semelhantes, exceto por um risco ligeiramente aumentado entre as usuárias de anticoncepcionais orais apenas com progestágeno ( $\mathrm{OR}$ 1,83, Intervalo de confiança - IC 95\% 0,63-5,34). A análise de outros antibióticos como controles negativos produziu resultados próximos da unidade (ORs variando de o,83 a I,13) (POTTEGARD ET AL,. 2018).

Segundo Pottegård et al,. Foi concluído que os achados não implicaram em uma associação entre o uso de dicloxacilina e o risco de falha dos anticoncepcionais orais e por último, apesar de a administração concomitante de dicloxacilina resultar em um aumento aproximado de duas vezes na atividade do $\mathrm{CYP}_{3} \mathrm{~A}_{4}$, isso pode não ser suficiente para reduzir o efeito da contracepção oral em uma extensão clinicamente importante.

Após realizada esta revisão bibliográfica, pode-se ressaltar que não são todos os antibióticos que inibem o efeito do anticoncepcional, principalmente antibióticos que não são indutores do citocromo $\mathrm{P} 450$, sendo assim, os antibióticos indutores do citocromo $\mathrm{P}_{450}$, principalmente a rifampicina, os maiores inibidores do efeito dos anticoncepcionais. $O$ ideal é que a paciente leia a bula e veja se aquele antibiótico tem essa capacidade de interagir com o anticoncepcional.

Embora a Rifampicina seja o único antibiótico de interação comprovada cientificamente, outros antibióticos foram citados em estudos, por apresentarem comportamento farmacológico de interação com os contraceptivos, porém não tão agressivo a ponto de levar as usuárias de C.O a uma falha do mesmo. Considerando um crescente número de novos antibióticos lançados no mercado, ainda há muito a se estudar, para poderem relacionar todas as interações que ocorrerem com o uso combinado de anticoncepcionais e antibióticos.

\section{REFERÊNCIAS}

Barry D Dickinson, Roy D Altman, Nancy H Nielsen, Melvyn L Sterling, Drug interactions between oral contraceptives and antibiotics, Obstetrics \& Gynecology, Volume 98, Issue 5, Part I, 200I, Pages 853-86o, ISSN 0029-7844, https://doi.org/ro.1016/Soo29-7844(or)or532-o.

Benet LZ, Kroetz DL, Sheiner LB. Farmacocinética A dinâmica da absorção, distribuição e 
eliminação dos fármacos. In: Hardman JG, Limbird LE, Molinoff PB, Ruddon RW, Gilman AG. Goodman \& Gilman as bases farmacológicas da terapêutica. 9a ed. New York: McGrawHill; 1996. p. 3-20.

Blode H, Zeun S, Parke S, Zimmermann T, Rohde B, Mellinger U, Kunz M. Evaluation of the effects of rifampicin, ketoconazole and erythromycin on the steady-state pharmacokinetics of the components of a novel oral contraceptive containing estradiol valerate and dienogest in healthy postmenopausal women. Contraception. 2012 Oct;86(4):33744. doi: Io.IoI6/j.contraception.2012.or.oro. Epub 2012 Mar 23. PMID: 22445438.

CORREAA, E.M.C.; ANDRADE, E.D.; RANALI, J. Efeito dos antimicrobianos sobre a eficácia dos contraceptivos orais. Rev Odontol Univ São Paulo, v. I2, n. 3, p. 237-240, jul./set.1998. Disponivel em http://www.scielo.br/scielo.php?script=sci_arttext\&pid=SoI0306631998000300007. Acesso 05/09/2021

Koopmans PC, Bos JH, de Jong van den Berg LT. Are antibiotics related to oral combination contraceptive failures in the Netherlands? A case-crossover study. Pharmacoepidemiol Drug Saf. 2012 Aug;2I(8):865-71. doi: 10.1002/pds.3267. Epub 2012 May 2. PMID: 22553004.

MARTINS, Nayana Bandeira et al. Análise de prescrição médica de antibióticos de uma farmácia comercial do município de Imperatriz-MA. Revista Científica do ITPAC, v. 7, n. 4, p. I-9, 20I4.

Masters KP, Carr BM. Survey of pharmacists and physicians on drug interactions between combined oral contraceptives and broad-spectrum antibiotics. Pharm Pract (Granada). 2009 Jul;7(3):139-44. doi: 10.4321/si886-36552009000300002. Epub 2009 Mar 15. PMID: 25143790; PMCID: $\mathrm{PMC}_{4139044 .}$

MENDONÇA, Deborah Soraia Brandao; RODRIGUES, Rafael Luiz Araújo. Interações Medicamentosas entre Antibióticos e Anticoncepcionais, presentes em Prescrições Médicas. ID on line REVISTA DE PSICOLOGIA, v. II, n. 35, p. 67-83, 2017. 
Pottegård A, Broe A, Stage TB, Brøsen K, Hallas J, Damkier P. Use of Dicloxacillin and Risk of Pregnancy among Users of Oral Contraceptives. Basic Clin Pharmacol Toxicol. 2018 Sep;123(3):288-293. doi: Io.11II/bcpt.1300o. Epub 2018 Apr I6. PMID: 29504695.

RAMOS, Fernanda Irene da Silva. Análise histórica das políticas de planejamento familiar no Brasil. 2008.

SOUZA, Lígia Kobelus de. Interação medicamentosa entre anticoncepcionais orais hormonais combinados e antibióticos. 2015.

Taylor J, Pemberton MN. Antibiotics and oral contraceptives: new considerations for dental practice. Br Dent J. 2012 May 25;212(I0):48I-3. doi: Io.1038/sj.bdj.2012.4I4. PMID: 22627223.

Toh, S., Mitchell, A. A., Anderka, M., de Jong-van den Berg, L. T., Hernández-Díaz, S., \& National Birth Defects Prevention Study (20II). Antibiotics and oral contraceptive failure - a case-crossover study. Contraception, 83(5),

https://doi.org/ıo.ıor6/j.contraception.2010.08.020

Townsend R, Dietz A, Hale C, Akhtar S, Kowalski D, Lademacher C, Lasseter K, Pearlman H, Rammelsberg D, Schmitt-Hoffmann A, Yamazaki T, Desai A. Pharmacokinetic Evaluation of $\mathrm{CYP}_{3} \mathrm{~A}_{4}-$ Mediated Drug-Drug Interactions of Isavuconazole With Rifampin, Ketoconazole, Midazolam, and Ethinyl Estradiol/Norethindrone in Healthy Adults. Clin Pharmacol Drug Dev. 2017 Jan;6(I):44-53. doi: I0.1002/cpdd.285. Epub 2016 Jul 25. PMID: 2727346r; PMCID: PMC5298035.

TURCATO, Thamires CC; CORREA, Milena A. Tonon. Interação medicamentosa pertinente a fármacos antibióticos e agentes anticoncepcionais femininos. 2016.

Wiesinger H, Klein S, Rottmann A, Nowotny B, Riecke K, Gashaw I, Brudny-Klöppel M, Fricke R, Höchel J, Friedrich C. The Effects of Weak and Strong CYP3A Induction by 
Rifampicin on the Pharmacokinetics of Five Progestins and Ethinylestradiol Compared to Midazolam. Clin Pharmacol Ther. 2020 Oct;108(4):798-807. doi: 10.1002/cpt.1848. Epub 2020 May II. PMID: 32275771; PMCID: PMC7540325. 